\title{
УСПЕШНОЕ ЛЕЧЕНИЕ ТЕРИПАРАТИДОМ ТЯЖЕЛОГО ОСТЕОПОРОЗА СМЕШАННОГО ГEHE3A
}

\author{
Бабарина М.Б., Пржиялковская Е.Г., Ульянова И.Н.
}

\author{
ФГБУ «НМИЦ эндокринологии» Минздрава России, Москва
}

Пациентка Т. впервые обратилась в НМИЦ эндокринологии в марте 2017 года в возрасте 66 лет с жалобами на выраженные боли в грудном и поясничном отделах позвоночника, снижение в росте с молодости на 15 см, одышку при ходьбе, онемение верхних конечностях, боли в коленных суставах.

Из анамнеза: ранняя менопауза с 45 лет, в 2007 году перенесла переломы ребер при минимальной травме. В августе 2016 года получала терапию ГКС в парентеральной форме, далее 6 месяцев принимала преднизолон перорально в дозе 15 мг в сутки. Из сопутствующей патологии: ХОБЛ, гормоно-зависимая форма БА, ГТЭ по поводу МУЗ, эутиреоз. Наличие СД, МКБ, ЯБ отрицала.

При осмотре: рост 140 см, вес 38 кг, ИМТ 19,4кг/м², выраженный кифоз грудного отдела позвоночника, укорочение туловища по сравнению с длиной нижних конечностей, уменьшение расстояния между реберными дугами и гребнями подвздошных костей, появление расстояния между стеной и затылком при измерении роста.

По МРТ позвоночника от января 2017- множественные компрессионные переломы (C4, C6, Th5, Th11, L2, L5), признаки отека костного мозга на уровне Th9, L2-L5. По данным KT органов грудной клетки- клиновидная деформация Th5, Th7, Th11, Th12, L2. В анализах крови показатели кальций-фосфорного обмена и креатинина в пределах нормы, ПтГ 34,6 пг/мл, бета-кросслапс 0,06(0-1,008) нг/мл, ОК 33,67 (11-43) нг/мл, ТТГ 2,71 Мед/л, анализ на миеломную болезнь - отрицательный. У пациентки выявлен дефицит витамина D (11 нг/мл), рекомендована терапия колекальциферолом и препаратами кальция. По данным денситометрии: снижение МПК по Т-критерию до -5.2SD в позвоночнике (L1-L4), до -3.8SD бедре в целом, до -3.5SD в шейке бедра. Установлен диагноз «Остеопороз смешанного генеза (постменопаузальный, глюкокортикоидный, на фоне дефицита веса и дефицита витамина D) тяжелого течения с множественными компрессионными переломами позвоночника с очень высоким риском переломов». В качестве антиостеопоротической терапии пациентке назначен деносумаб 60 мг подкожно 1 раз в 6 месяцев.

На момент повторного обращения в августе 2018 года в анализах крови: показатели кальций фосфорного обмена в пределах нормы, бета-кросслапс 0.17(0-1,008)нг/мл, ОK 0,55(11-43) нг/мл, витамин D 53 нг/мл. По денситометрии: L1-L4 -4,6SD, бедро Total -3,5SD, бедро Neck -3,5SD по T-критерию. Учитывая сохранение выраженной потери МПК, наличие множественных компрессионных переломов, рекомендовано по жизненным показаниям дополнительно терапия терипаратидом 20 мкг подкожно ежедневно, колекальциферол заменен на альфакальцидол, продолжить прием препаратов кальция. С декабря 2018 года пациентка начала лечение терипаратидом, переносила удовлетворительно. По анализам крови на бета кросслапс и ОК через 3 месяца выявлен прирост этих показателей, что свидетельствует об эффективности проводимого лечения. Пациентка отмечала уменьшение болей в спине, расширение физической нагрузки, новых переломов зафиксировано не было. По денситометрии через год : L1-L4 -3,9SD по Т-критерию, бедро Total -3,5SD, бедро Neck=-3,4SD.

Таким образом, терипаратид в комбинации с деносумабом показал высокую эффективность в лечении тяжелого остеопороза, обеспечив прибавку МПК и предупреждение новых низкотравматических переломов позвонков. 